\title{
TILLANDSIA MAGNISPICA (BROMELIACEAE: TILLANDSIOIDEAE), A NEW SPECIES FROM OAXACA, MEXICO
}

\author{
Adolfo Espejo-Serna y Ana Rosa López-Ferrari
}

Universidad Autónoma Metropolitana-lztapalapa, División de Ciencias Biológicas y de la Salud, Departamento de Biología, Herbario Metropolitano, 09340 México, D.F. México. aes@xanum.uam.mx

\begin{abstract}
Tillandsia magnispica Espejo \& López-Ferrari, known only from the state of Oaxaca, Mexico, in the municipalities of San Pedro el Alto and San Pedro Pochutla, is described and illustrated. The new taxon is compared with T. jaliscomonticola Matuda, a morphologically similar species.
\end{abstract}

Key words: Bromeliaceae, Mexico, Oaxaca, Tillandsia.

\section{RESUMEN}

Se describe e ilustra Tillandsia magnispica Espejo \& López-Ferrari, conocida sólo del estado de Oaxaca, México, en los municipios de San Pedro el Alto y San Pedro Pochutla. La nueva especie se compara con T. jaliscomonticola Matuda, con la cual tiene afinidades morfológicas.

Palabras clave: Bromeliaceae, México, Oaxaca, Tillandsia.

As a result of our explorations in the field and the revision of herbarium material in the main Mexican and foreign institutional herbaria, in order to complete the Flora Bromeliológica Mexicana, we detected material from one undescribed species of the genus Tillandsia that we propose here as: 
Tillandsia magnispica Espejo et López-Ferrari, sp. nov. Figs. 1, 3B

Herba epiphytica, acaulis, rosulata, florens usque $80 \mathrm{~cm}$ alta. Folia numerosa, vaginae distinctae, ellipticae, 7-10 cm longae, 4-5 cm latae; laminae anguste triangulari-lanceolatae, $37-55 \mathrm{~cm}$ longae, 1.3-1.7 cm latae, involutae, distaliter arcuatae; scapus inflorescentiae erectus, teres, $29-50 \mathrm{~cm}$ longus; inflorescentia simplicissima; spica anguste elliptica, complanata, $18-25 \mathrm{~cm}$ longa, $3.5-4.5 \mathrm{~cm}$ lata; bracteae florales distichae, imbricatae, late ovatae, $5-5.5 \mathrm{~cm}$ longae, $4-4.5 \mathrm{~cm}$ latae, carinatae, supera virides, basalia incarnatae, nervatae; petala $7-8 \mathrm{~cm}$ longa, ca. $1 \mathrm{~cm}$ lata, violacea; stamina subaequalia, exserta, stylus exsertus.

Plants stemless, epiphytic, flowering up to $80 \mathrm{~cm}$ high; leaves numerous, forming a tank rosette ca. $40 \mathrm{~cm}$ in diameter; leaf sheaths elliptic, 7-10 cm long, $4-5 \mathrm{~cm}$ wide, glabrous and dark brown adaxially, glabrous and light brown abaxially; leaf blades narrowly triangular to lanceolate, $37-55 \mathrm{~cm}$ long, $1.3-1.7 \mathrm{~cm}$ wide at the widest part, coriaceous, lepidote, tapering to a long filiform and acuminate apex, margins involute; inflorescence erect, simple, to $75 \mathrm{~cm}$ long; scape $29-50 \mathrm{~cm}$ long, erect, stout, ca. $1 \mathrm{~cm}$ in diameter, scape bracts polystichous, imbricate, rose-coloured, the lower foliaceous, the blades similar to the leaves, the upper bladeless or with much reduced blades, linear-subulate, the sheaths ovate, to $5 \mathrm{~cm}$ long and $3 \mathrm{~cm}$ wide, concealing the scape; spike erect, narrowly elliptic to oblong, $18-25 \mathrm{~cm}$ long, $3.5-4.5 \mathrm{~cm}$ wide, complanate, up to 28 flowered, larger than the leaves, floral bracts distichous, imbricate, longer than the sepals, glabrous, the basal ones rose-coloured, the upper ones bright green, marginally wine-coloured, widely ovate, conduplicate, carinate, acuminate, with a pungent apex, conspicuously nerved when dried, $5-5.5 \mathrm{~cm}$ long, 4-4.5 cm wide; flowers, erect, sessile, successive; sepals elliptic to oblong, glabrous, acute, the posterior ones carinate and shortly connate at the base, pale green, 3.4-4 cm long, 1-1.2 cm wide; petals narrowly oblong to spatulate, glabrous, obtuse, free, violet at the upper half, white at the base, $7-8 \mathrm{~cm}$ long, $\mathrm{ca} .1 \mathrm{~cm}$ wide, stamens subaequal, filaments linear, $8.7-9 \mathrm{~cm}$ long, violet and complanate at apical end, white at the base, anthers oblong, yellow, 4.5-5 mm long; ovary ovoid, green, ca. $7 \mathrm{~mm}$ long, $5 \mathrm{~mm}$ in diameter; style white, $8.7-9.5 \mathrm{~cm}$ long; stigma white, trilobulate, spiral conduplicate (Brown \& Gilmartin, 1984), 4.5-5 $\mathrm{mm}$ in diameter; fruit not seen.

Type: México, Oaxaca, distrito de Pochutla, municipio de San Pedro Pochutla, alrededores de Toltepec, ca. $4.5 \mathrm{~km}$ después de San José Chacalapa, carretera Pochutla-Miahuatlán, 1551'47" N; 96²7'58" W, 285 m s.n.m., selva mediana, 

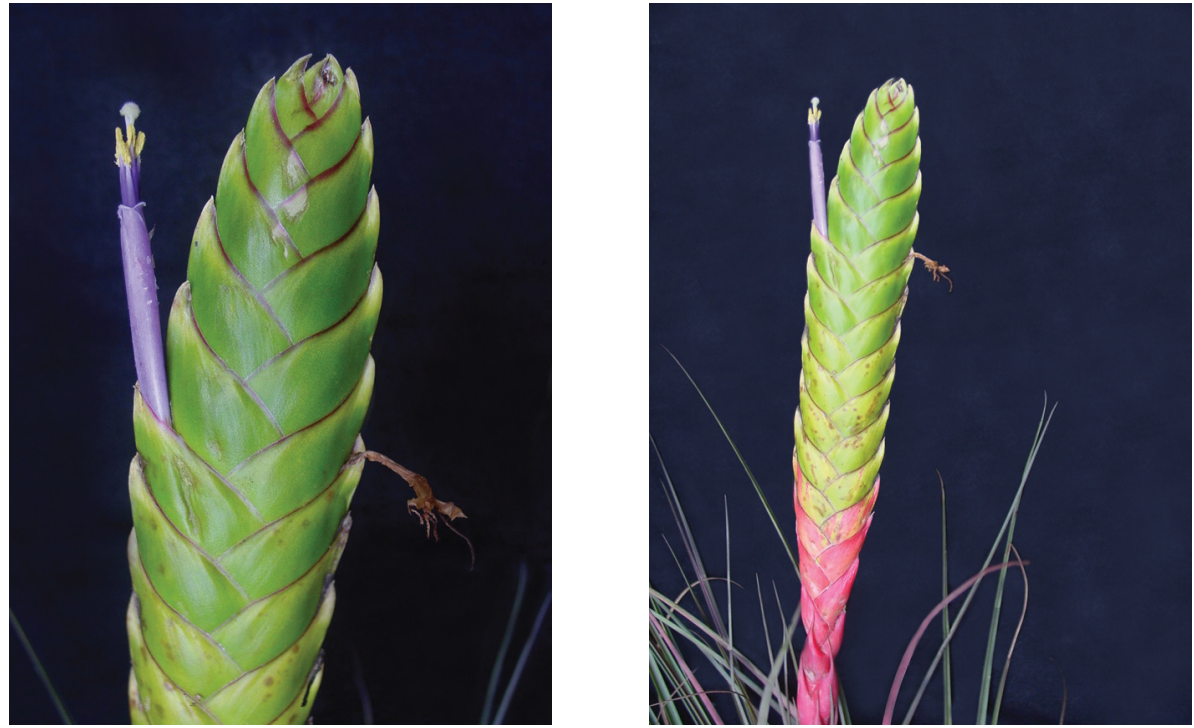

Fig. 1. Tillandsia magnispica Espejo \& López-Ferrari (A. Espejo et al. 6312).
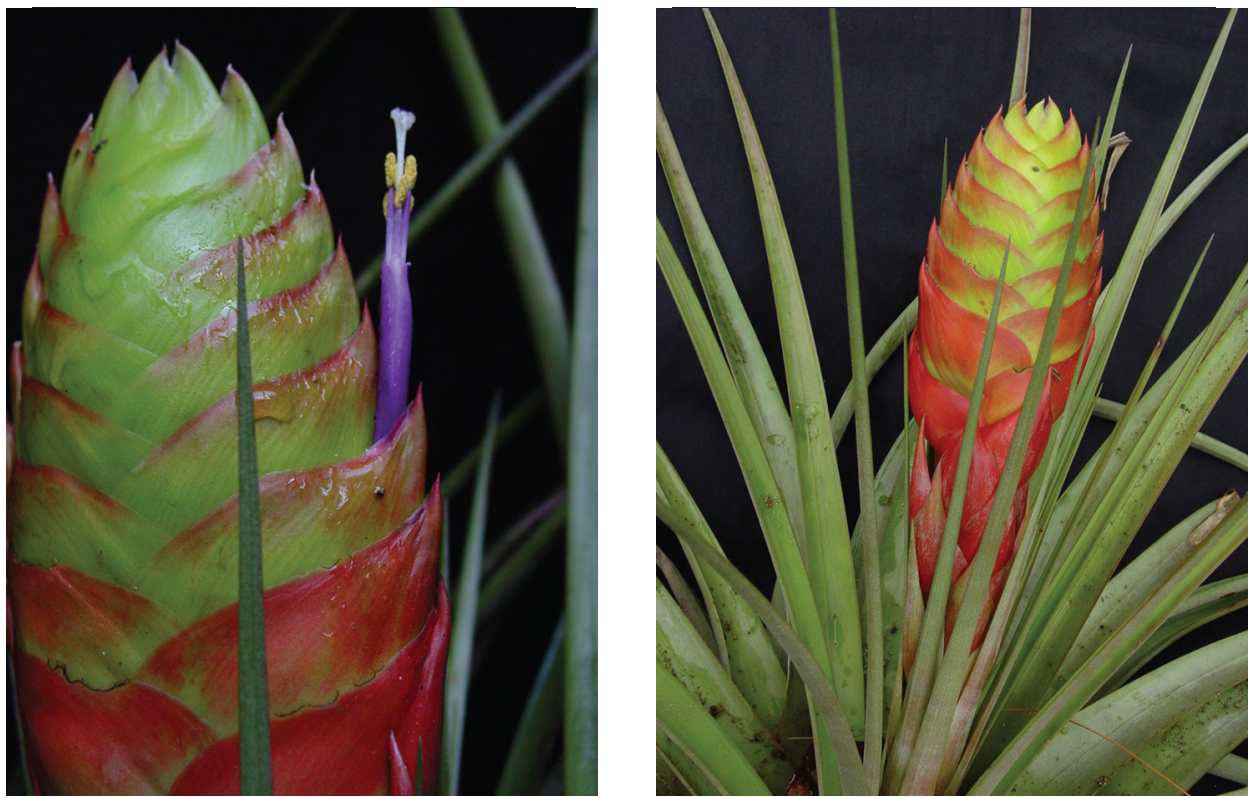

Fig. 2. Tillandsia jaliscomonticola Matuda (J. Ceja et al. 1473). 
10.VIII.2001, A. Espejo, A. R. López-Ferrari, J. Ceja y A. Mendoza R. 6312 (Holotype (in three sheets): UAMIZ; Isotype IEB).

Paratypes: Mexico, Oaxaca, distrito de Pochutla, municipio de San Pedro Pochutla, N of Pochutla, 1981, C. S. Gardner 1445 (SEL, US, line drawing by Gardner, 1982); distrito de Pochutla, municipio de San Pedro el Alto, $18 \mathrm{~km}$ al S de San Miguel Suchistepec, sobre la carretera a Pochutla, 1700 m s.n.m. Bosque de Pinus

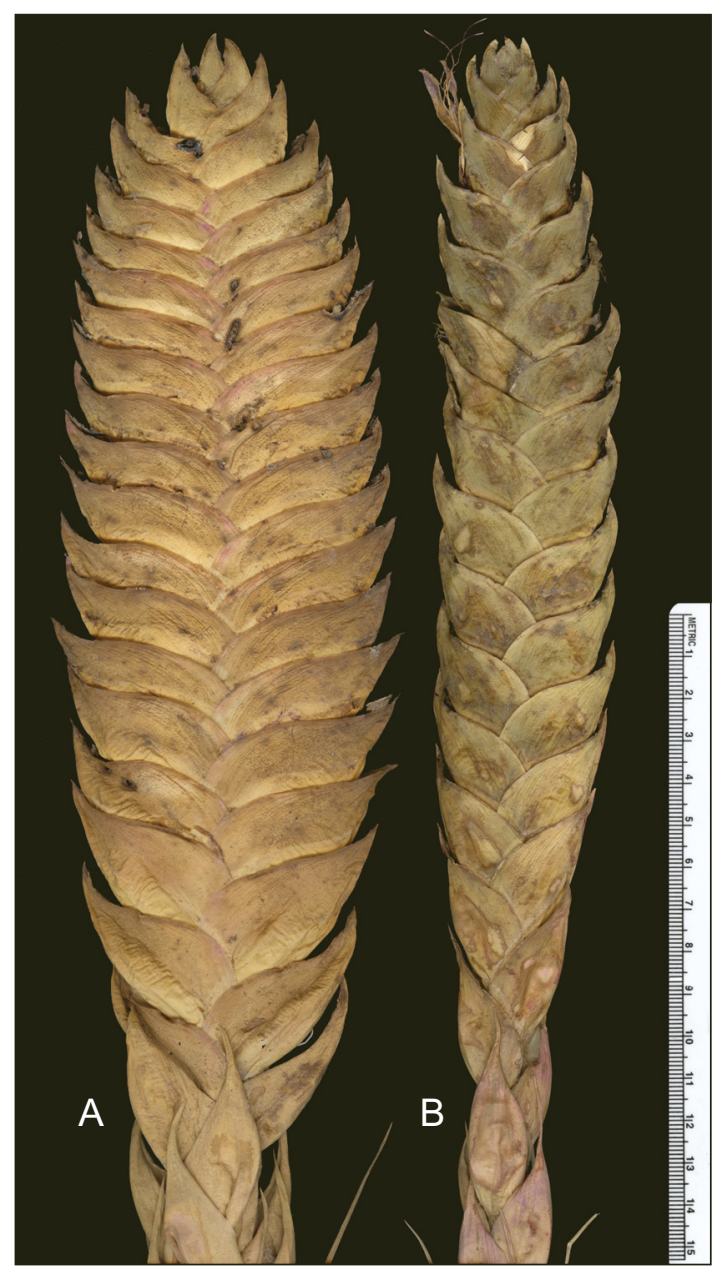

Fig. 3. Dried spikes. A. Tillandsia jaliscomonticola Matuda (J. Ceja et al. 1473 (UAMIZ)); B. Tillandsia magnispica Espejo \& López-Ferrari (A. Espejo et al. 6312 (UAMIZ)). 
oocarpa sobre ladera metamórfica, 22.IX.1965, J. Rzedowski 20648 ENCB(x3), LL, MICH, TEX).

Tillandsia magnispica is endemic to Oaxaca (Espejo-Serna et al., 2007 p. 138), and known only from the Municipalities of San Pedro Pochutla and San Pedro el Alto, District of Pochutla (Fig. 4).

The material of the new species has been identified by some authors (Gardner, 1982, pp. 145-146; McVaugh, 1989, p. 58) as Tillandsia jaliscomonticola Matuda (Figs. 2, 3A), a taxon only known from Colima, Jalisco, and Michoacán (Espejo et al., 2004, p. 63) (Fig. 4 and Appendix), however the differences between the two taxa showed in the table 1 and figures 1,2 , and 3, are conspicuous.

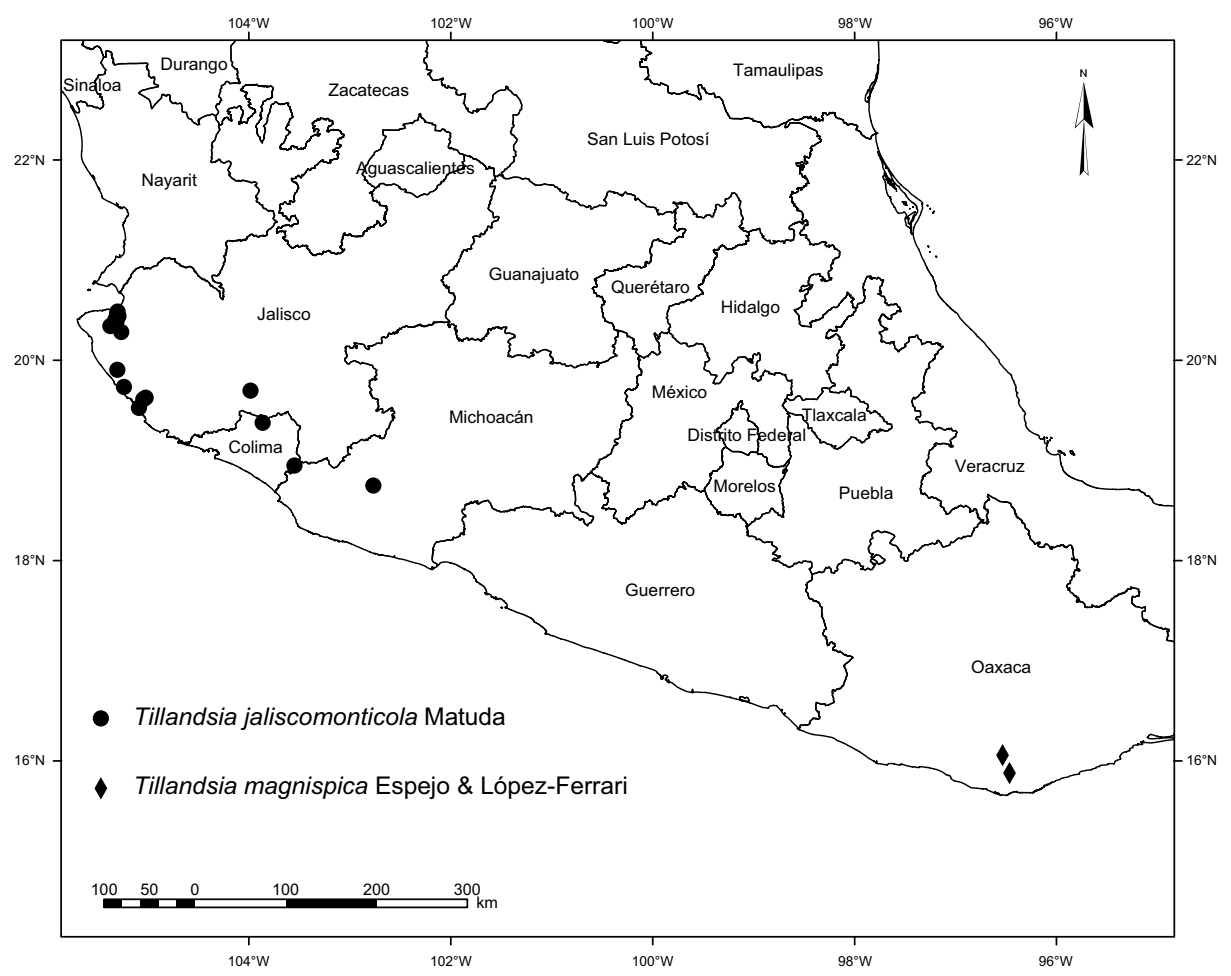

Fig. 4. Distribution map of Tillandsia magnispica Espejo \& López-Ferrari, and $T$. jaliscomonticola Matuda. 
Table 1. Comparative characteristics of T. magnispica and T. jaliscomonticola.

\begin{tabular}{lll}
\hline & T. magnispica & T. jaliscomonticola \\
\hline foliar sheats dimensions & $7-10 \mathrm{~cm}$ long, $4-5 \mathrm{~cm}$ wide & $11.5-15 \mathrm{~cm}$ long, $5.5-6 \mathrm{~cm}$ wide \\
spike number & 1 & $1-3$ \\
spike width & $3.5-4.5 \mathrm{~cm}$ & $6-8.2 \mathrm{~cm}$ \\
petals length & $7-8 \mathrm{~cm}$ long & $6-7 \mathrm{~cm}$ long \\
stamens & subaequal & unaequal \\
geographical distribution & Oaxaca & Colima, Jalisco and Michoacán \\
\hline
\end{tabular}

\section{ACKNOWLEDGMENTS}

We thank the following herbaria for providing specimens and data used in this work: ENCB, IEB, LL, MEXU, MICH, MO, SEL, TEX, UAMIZ and US; Jacqueline Ceja-Romero and Aniceto Mendoza-Ruiz for their invaluable help with field work; and Nancy Martínez-Correa for the elaboration of the map.

\section{LITERATURE CITED}

Brown, G. K. \& A. J. Gilmartin. 1984. Stigma structure and variation in Bromeliaceae neglected taxonomic characters. Brittonia 36: 364-374.

Espejo, A., A. R. López-Ferrari, I. Ramírez-Morillo, B. K. Holst, H. E. Luther \& W. Till. 2004. Checklist of Mexican Bromeliaceae with notes on species distribution and levels of endemism. Selbyana 25: 33-86.

Espejo-Serna, A., A. R. López-Ferrari, N. Martínez-Correa \& V. A. Pulido-Espaza. 2007. Bromeliad flora of Oaxaca, Mexico: richness and distribution. Acta Bot. Mex. 81: 71-147.

Gardner, C. S. 1982. A systematic study of Tillandsia subgenus Tillandsia.Ph. D. Dissertation. Texas A \& M University, College Station. Corpus Christi. 305 pp.

McVaugh, R. 1989. Bromeliaceae. In: Anderson, W. R. (ed.). Flora Novo-Galiciana. The University of Michigan Herbarium. Ann Arbor. vol. 15 pp. 4-79. 
Espejo-Serna y López-Ferrari: Tillandsia magnispica, a new species from Oaxaca, México

\section{APPENDIX}

\section{Tillandsia jaliscomonticola exsiccata}

Colima, municipio de Ixtlahuacán: 26 de julio, a $12 \mathrm{~km}$ al E de Agua de la Virgen, 1857'2" N, 10332'30" W, 23.IX.1983, E. J. Lott, E. Martínez S. y J. A. S. Magallanes $1925 A$ (MEXU, MICH, MO); municipio de Minatitlán: Cerro Grande, brecha de Juloapan a Lagunitas, después del campo 4, 19²2'40" N, 103 $51^{\circ} 30^{\prime \prime} \mathrm{W}$, bosque tropical caducifolio, 17.X.1990, M. Cházaro B., M. Huerta M., C. Ortiz A. y J. Gómez H. 6454 (IEB). Jalisco, municipio de Cabo Corrientes: near Puerto Vallarta, IX.1994, W. Berg s. $n$. (SEL); sierra El Tuiz (Tuito), 27.X.1974, C. R. Beutelspacher sub E. Matuda 38657 (MEXUx2); ca. $11 \mathrm{~km}$ después de El Tuito, rumbo a Puerto Vallarta, 12.IV.2003, J. Ceja, A. Espejo, A. R. LópezFerrari y A. Mendoza R. 1438 (UAMIZx5); ca. 0.5 km después de Pedro Moreno, rumbo a

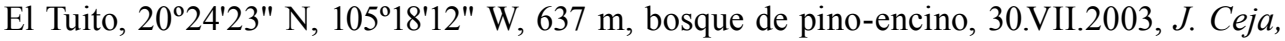
A. Espejo, A. R. López-Ferrari, A. Mendoza R. e I. Ramírez M. 1473 (UAMIZx5); along the highway in the gorge of the río Horcones, south of Puerto Vallarta, about $22.5 \mathrm{~km}$ from Puerto Vallarta, 5.XI.1971, J. V. A. Dieterle 4067 (ENCB, MEXU, MICH); aproximadamente $1 \mathrm{~km}$ sobre la brecha a Coyonzalo, a partir del km 187 de la carretera Puerto Vallarta - El Tuito, 2.IV.1993, A. R. López-Ferrari y A. Espejo 1771 (UAMIZx3); $6.1 \mathrm{~km}$ al W de El Tuito, sobre el camino a Ixtlahuahuey, 17.X.1985, P. Magaña 353 (MEXUx2); $10 \mathrm{~km}$ al N de El Tuito, $115 \mathrm{~km}$ al N de la estación de biología Chamela, sobre la carretera Barra de Navidad - Puerto Vallarta, 18.I.1985, P. Magaña y E. J. Lott 42 (ENCB, MEXU, MO); $32 \mathrm{~km}$ al S de Puerto Vallarta sobre la carretera que va a Barra de Navidad, 1.IX.1985, P. Magaña, $R$. Ayala y G. Ayala 279 (ENCB, MEXUx2), municipio de La Huerta, estación de biología Chamela, vereda Calandria, $100 \mathrm{~m}$ antes de la estación meteorológica, 19.I.1985, P. Magaña y E. J. Lott 46 (ENCB), $9.4 \mathrm{~km}$ del camino Juan Gil Preciado - Los Ranchitos - Nacastillo, 15.X.1985, P. Magaña, A. Solís, E. J. Lott y G. Ayala 322 (MEXU); $6.1 \mathrm{~km}$ del camino Juan Gil Preciado - Los Ranchitos - Nacastillo, 15.X.1985, P. Magaña, A. Solís, E. J. Lott y G. Ayala 333 (ENCB, LL, MEXUx2); municipio de Tomatlán: $47 \mathrm{~km}$ al $\mathrm{N}$ de la estación de biología Chamela, sobre la carretera Barra de Navidad - Puerto Vallarta, 30.IV.1985, P. Magaña y E. J. Lott 239 (MEXU); near the new road 4-22 km northwest of río San Nicolás and 20-40 km southeast of Tomatlán, 11.XII.1970, R. McVaugh 25251 (MICH); municipio de Tuxcacuesco: Tuxcacuesco, 13.XI.1943, E. A. Anderson s. n. (MO); sin localidad, II.1992, A. Lau Jr. sub H. E. Luther s. n. (SEL). MichOACÁn, municipio de Aguililla, $4 \mathrm{~km}$ al NO de Aguililla, $18^{\circ} 45^{\prime} \mathrm{N}, 12^{\circ} 45^{\prime} 45^{\prime \prime} \mathrm{W}, 950 \mathrm{~m}$, selva baja caducifolia, 9.IV.1985, J. C. Soto N., A. Román de Soto y F. Soto R. 8067 (MEXU). 
